\title{
Donepezil and Related Cholinesterase Inhibitors as Mood and Behavioral Controlling Agents
}

\author{
Tal Burt, M D
}

\begin{abstract}
Address
Medical Director, D epression/A nxiety W orldwide Team, Pfizer Inc. 235 East 42nd Street, 235/10/29, N ew York, NY 10023, USA.

E-mail: tal.burt@ pfizer.com
\end{abstract}

Current Psychiatry Reports 2000, 2:473-478

Current Science Inc. ISSN 1523-3812

Copyright $\odot 2000$ by Current Science Inc.

A cetylcholinesterase inhibitors ( $\mathrm{ChEls}$ ) enhance neuronal transmission by increasing the availability of acetylcholine in muscarinic and nicotinic receptors. This effect is believed to be responsible for the beneficial and protective effects of $C h E l s$ on cognition in patients with Alzheimer's disease (AD). Effects of ChEls on mood and behavior have also been reported. Earlier observations were limited by the exclusive availability of intraveno us forms of administration, the short half-life of the formulations, and the high frequency of peripheral side effects. The introduction, in recent years, of better tolerated and less invasive compounds has rekindled the interest in cholinergic central nervous system mechanisms and has given rise to studies in areas other than cognition. The $\mathrm{ChEl}$ donepezil has been involved in the largest number of studies and positive reports. Preliminary observations suggest the possible value of $\mathrm{ChEls}$ in the management of behavioral dysregulation, apathy, irritability, psychosis, depression, mania, tics, and delirium and in the diagnosis of depression, panic, and personality disorders.

\section{Introduction}

Acetylcholine is a neurotransmitter distributed extensively throughout the central nervous system (CNS). It modulates neuronal activity through agonist effects on muscarinic and nicotinic receptors. Acetylcholinesterase inhibitors (ChEls) enhance neuronal transmission by preventing the hydrolysis of acetylcholine by the enzyme acetylcholinesterase, thus increasing the availability of acetylcholine in muscarinic and nicotinic receptors.

Cholinergic neurons demonstrate extensive distribution throughout the CNS and the peripheral nervous system. In the CNS they are believed to be associated with cognitive functions. One of the hypotheses of the origin of
Alzheimer's disease (AD) postulates loss of cholinergic neurons as the primary cause. ChEls have been used successfully in the improvement of cognition and slowing of cognitive deterioration in $A D$ patients. It is believed that increased cholinergic tone is responsible for the beneficial effect of ChEls on cognition.

In addition to effects on cognition, sporadic reports, mainly in the latter half of the 20th century, havesuggested that cholinergic mechanisms may have a role in the modulation of human mood and behavior. Anecdotal reports date back to as early as 1889 when Willoughby [1] reported the resolution of manic symptoms following intravenous administration of pilocarpine. In the 1950s use of cholinesterase inhibitors in the form of organophosphate insecticides was found to be associated with an unusual frequency of depressive and psychotic symptoms $[2,3,4]$. In 1972 Janowsky et al. [5,6] published a report describing the resolution of manic symptoms following intravenous administration of the ChEl physostigmineand postulated the cholinergic-adrenergic hypothesis of mania and depression. According to the hypothesis, cholinergic and adrenergic tone had reciprocating and bal ancing effects on mood, adrenergic tone being manicogenic or antidepressant, and cholinergic tone being depressogenic or antimanic. In further support of the hypothesis were observations that cholinergic antagonist such as scopolamine may induce euphoria, talkativeness, difficulties in concentration, and flight of ideas [7], and that cholinomimetics may cause changes in sleep patterns (eg, decrease REM latency) and hormone levels (eg, adrenocorticotropic hormone [ACTH], cortisol) similar to those found in patients with major depression $[8,9]$.

Critics of the cholinergic-adrenergic hypothesis daimed that the behavioral effects observed with ChEls are secondary and nonspecific reaction to the wide range of unpleasant peripheral side effects they generate and the stress and mal aise that ensue $[10,11]$. The short half-life (10-20 minutes) of physostigmine, the presence of central as well as peripheral effects, and the obligatory intravenous administration, were considered significant obstacles to the testing of the effects of ChEls on mood and behavior [12].

In the past decade, the advent of oral ly administered ChEls, with longer half-life and a moretolerable side-effect 
profile, devel oped mainly for the treatment of cognitive deficits in AD, has led to an increasing number of reports on the effects of ChEls in mood and behavior. This review focuses mainly on advancements madein the field over the past 2 to 3 years.

\section{Properties of Cholinesterase Inhibitors}

As a class ChEls share a propensity for certain side effects. These include nausea, vomiting, anorexia, diarrhea, insomnia, fatigue, muscle cramps, and headache. The frequency and severity of these side effects vary substantially from compound to compound and appear to be related to the rate at which the dose of the compound is increased.

\section{Donepezil}

Donepezil hydrochloride is a reversible inhibitor of the enzyme acetylcholinesterase The elimination half-life of donepezil is about 70 hours and pharmacokinetics are linear. It is approximately $96 \%$ bound to plasma proteins, al though it was not found to have any significant effects on the binding of other highly protein bound drugs, including warfarin, digoxin, and furosemide. Nor do these drugs significantly affect donepezil. Donepezil is both excreted in the urine intact and extensively metabolized by CYP 450 isoenzymes 2D6 and 3A4 and by undergoing glucuronidation. Donepezil has greater selectivity for acetylcholinesterase (AChE), the type of ChE prevalent in the CNS, than it has for butylcholinesterase (BChE), the type of ChE which is primarily active outside of the blood brain barrier. The AChE:BChE ratio of donepezil (1200:1) is the highest available in this class of agents. This leads to increased central versus peripheral cholinergic specificity and is believed to be responsible for the favorable side effect profile of donepezil [13].

\section{Tacrine}

Tacrine hydrochloride is a reversible inhibitor of the enzyme acetylcholinesterase Tacrine inhibits both AChE and BChE; but it is more selective for AChE than physostigmine. Tacrine undergoes extensi ve hepatic metabolism by the cytochrome P450 1A2 isoenzyme to at least three metabolites. The major metabolite, 1-hydroxy-tacrine (velnacrine), is active Metabolites are excreted in the urine Elimination half-life is between 1.5 and 4 hours. Tacrine is known to produce significant elevations in liver enzymes, notably the transaminases. These el evations are generally reversible and tend to behigher in women.

\section{Rivastigmine}

Rivasti gmi ne is a carbamate-type pseudo-i rreversi ble ChEl. Rivastigmine is not metabolized by the hepatic cytochrome P450 oxidative enzyme system and it exhibits approximately $40 \%$ protein-binding. Thus, drug interactions with rivastigmine are unlikely. Ri vastigmine is rapidly and extensi vely metabolized primarily at CNS receptor sites via cholinesterase. The plasma half-lives of rivastigmine and the major metabolite are roughly 1 hour and 2 hours, respectively; however, the cholinesterase inhibition in theCNSIasts much longer (average 10 hours) than the short plasma half-life would predict. This is due to the fact that when rivastigmine's phenolic ZN N-666 metabolite is formed, it leaves behind a carbamate moiety that stays attached to the AChE receptor for up to 10 hours, which prevents the hydrolysis of ACh. Renal excretion of the metabolite is the major route of el imination.

\section{Metrifonate}

Metrifonate differs from other AChE inhibitors in that it is a prodrug that is nonenzymatically converted in vivo to the active moiety 2,2-dichlorovinyl dimethyl phosphate (DDVP). DDVP administered alone has a very short plasma elimination half-life, but small amounts released from metrifonate are sufficient to inhibit AChE activity in vivo. DDVP isan irreversibleinhibitor of $A C h E$ and activity is maintained for several weeks. It al so inhibits BChE. Metrifonate has been withdrawn from marketing consideration due to safety concerns.

\section{Galantamine}

Gal antamine is a reversible, competitive cholinesterase inhibitor. In addition, it al losterically modulates nicotinic acetylcholine receptors. Galantamine is approximately 50 times more effective against human AChE than against human BChE at therapeutic doses.

\section{Controlled Release Physostigmine}

A new extended-rel ease formulation of physostigmine salicylate that yields sustained blood levels, permitting twice-daily dosing. It inhibits both AChE and BChE. It is associated with high frequency of gastrointestinal side effects.

\section{Effect of Cholinesterase Inhibitors on Mood and Behavior in Alzheimer's Disease}

Most available cholinesterase inhibitors exhibit a similar beneficial effect on cognition in AD patients. Patients with $A D$, however, typically present with a variety of neuropsychiatric symptoms including depression, apathy, agitation, hallucinations and psychosis. In addition, patients' ability to perform activities of daily living (ADL) is a crucial factor in the assessment of their disability, the burden on caregivers, and whether they require placement in a nursing facility or not. As a consequence, studies of ChEls in AD started to include, in addition to measures of cognitive function, also measures of mood and behavior, such as the Neuropsychiatric Inventory (NPI) [14].

\section{Donepezil}

Donepezil was associated with the largest number of positive observations supporting a beneficial effect on mood 
and behavior in $A D$ patients. Weiner et al. [15] found that donepezil administration was associated with prevention of worsening depression and behavioral dysregulation over a 12-month period in AD patients. [15] Cummings et al. [16] reported that AD patients taking donepezil had lower lever of behavioral disturbances, were less threatening, and needed fewer sedatives than those not on donepezil as reported by caregivers after a 6 -month treatment period. In an open-label study of 2092 patients with mild-moderate $A D$ donepezil improved ADL, mood, social behavior, and disturbing behavior, as well as memory after 3 months when compared with baseline. Improvements were specifical ly reported in urinary incontinence, nighttime restlessness, irritability, aggressive behavior, stubbornness, wandering, and bowel control [17]. Hecker [18] reported improvement with donepezil in depression, anxiety, and apathy areas of the NPI when compared with placebo over 4 and 24 weeks in 191 moderate-severe AD patients. In a retrospective study in 86 AD patients Mega et al. [19] observed significant improvement from baseline in delusions, agitation, anxiety, disinhibition, and irritability in responders to donepezil. The behavioral changes were dose-dependent. They concluded that donepezil has psychotropic properties, and pretreatment behaviors help predict patients' responses to treatment. The same group also demonstrated that $A D$ patients that responded to donepezil with reduction of irritability, delusions, hallucinations, agitation, euphoria, and aberrant motor behavior, also demonstrated lower regional perfusion in the dorsolateral parietal, orbital frontal, and anterior cingulated bilaterally [20]. More recently they reported that $A D$ patients who responded to ChEl therapy had significantly more pretreatment irritability, disinhibition, and euphoria than nonresponders on the NPI, and significantly lower lateral orbital frontal $(P<0.00001)$ and dorsolateral frontal ( $P \varangle 0.0005$ ) perfusion bilaterally as evidenced by $99 \mathrm{~m}$ labelled D, L-hexamethyly-propylene amine oxime (99m Tem-HMPAO) single photon emission computed tomography. They concluded that a pretreatment orbitofrontal syndrome may predict behavioral response to ChEl therapy in AD [21•].

\section{Metrifonate in Alzheimer's disease}

Several studies have demonstrated the efficacy of metrifonate in improving neuropsychiatric symptoms in $A D$ patients. Kaufer [22 - ] reported beneficial effects of metrifonate in AD patients on the NPI total score, aberrant motor behavior, and in symptoms of depression, apathy and hallucinations when compared with placebo in a 26week trial. In a study by Morris et al. [23] metrifonate was associated with improvements in behavioral and global functioning as well as cognitive functioning in mildmoderate AD patients over a 36-week period when compared with placebo. Cummings et al. [24] and
Raskind et al. [25] reported similar results in mild-moderate $A D$ patients over a 6 -month period as measured by the NPI. In a negative study, Becker et al. [26] reported that al though metrifonate was associated with slowing the severity of cognitive symptoms in AD patients over a 6month period, it did not affect noncognitive behaviors measured by the AD Assessment Scale noncognitive subscale (ADAS-N), Global Improvement Scale, or the ADL Checklist.

\section{Other acetylcholinesterase inhibitors in Alzhei mer's disease}

Tacrine was associated with a higher percentage of patients exhibiting improvement on the Alzhei mer's Disease Assessment Scale(ADAS) noncognitiveitems: cooperation, delusions, and pacing, as well as improvement in cognition when compared with placebo in a 30-week trial [27].

Rivastigmine was associated with significant improvement in activities of daily living, and global evaluation ratings in addition to cognitive functioning when compared with placebo in mild-moderate $A D$ patients over a 6-month period. Twenty three percent of patients taking rivastigmine discontinued due to adverse events compared with $7 \%$ in the placebo group [28].

In a 5-month placebo-controlled study in mild to moderate AD patients, galantamine was associated with improvement in behavior and functioning as measured by the Clinician's Intervi ew-Based Impression of Change, Caregiver Input, and AD CooperativeStudy ADL Inventory, as well as improvement in cognition as measured by the AD Assessment Scale cognitive subscal e [29]. In another study in mild-moderate $A D$ patients, improvements in functioning and cognition were observed at 6 months on gal antamine versus placebo [30].

In two studies involving extended release physostigmine there was a significant improvement in global functioning as well as cognitive functioning when compared with placebo in mild-moderate $A D$ patients, but the treatment was al so associated with a high incidence of adverse events (in one study $78.6 \%$ of patients experienced nausea, $61.5 \%$ vomiting; in theother study $37.6 \%$ of patients discontinued the study due to adverse events) [31, 32].

In view of the beneficial effect of ChEls on cognition, and considering the relationship between cognition, mood, and behavior, on biologic, psychologic, and social levels, the primary effect of ChEls on mood and behavior needs to be established especially in patients with preexisting cognitive deficits. For example, improved cognition may in itself be responsible for improvement in mood and behavior: patients with impaired skills that are necessary for ADL and social integration may be observed to demonstrate less disruptive behavior and irritable mood simply because improved cognition has led to better social integration and reduced environmental stress. 


\section{Cholinesterase Inhibitors} in Mood Disorders and Schizophrenia

In both mood disorders and schizophrenia cognitive deficits are common as well as are behavioral and mood disturbances [33]. Whereas in mood disorders the primary focus was on the ability of cholinergic systems to normalize depression or mania, in schizophrenia the main focus was on the association of cognitive and functional deficits.

Burt et al. [34 - ] reported a case series of 11 patients with treatment-resistant bi polar disorder who received donepezil for a period of 6 weeks or more. Six patients had an improvement of two points or more on the Clinical Global Impression-Severity of IIIness (CGI-S) [35], a seven-point scale. Five of the patients that improved demonstrated improvement within the first 2 weeks. Of note, one of the patients suffered from bi polar depression and demonstrated a three-point improvement on the CGI-S. Improvement of depression with ChEls can be explained by the agonistic effect on nicotinic receptors. Nicotine may posses antidepressant effects as is suggested by the comorbidity of depression and nicotine dependence, as well as by the demonstrated efficacy of antidepressants in the treatment of nicotine dependence (eg, bupropion). If, in addition, ChEls exert antimanic effects as well, through agonist activity on muscarinic receptors, ChEls may be considered true bimodal mood-stabilizing agents.

In schizophrenia, Risch et al. [36] reported improvements in verbal fluency and attention in six patients on donepezil. Concurrent activation of the right dorsolateral prefrontal cortex and anterior cingulated on functional magnetic vesonance imaging was also observed. Bergman et al. [37] reported a casestudy of a patient with schizophrenia, resistant to clozapine, haloperidol, and fluphenazine, and partially responding to risperidone, that experienced improvement in psychotic symptoms and cognitive function within 4 weeks of starting donepezil. The patient maintai ned these improvements at a 6-month follow-up. Donepezil treatment was also associated with improved behavioral functioning and performance in neurocognitive test batteries in two treatment refractory patients with schizophrenia, in an open-label treatment [38]. Burke et al. [39] reported the resolution of postoperative visual hallucinations in a 74-year-old man after starting donepezil.

\section{Cholinesterase Inhibitors in Tourette's Disorder and Attention Deficit Hyperactivity Disorder}

Recent literature on tic disorders has focused on serotonin and dopamine, although reports of improvement in tics with nicotine treatment suggest a role for acetylcholine as well. Tacrine was associated with a significant ded ine in self-rated attentional deficits, a significant improvement in objective measures of sustained attention, and a nearsignificant decrease in examiner-rated tics in an open-label study involving six patients with Tourette's disorder [40].
Hoopes et al. [41] reported on two patients, 11 and 13 years old, both with Tourette's disorder and attention deficit hyperactivity disorder (ADHD), resistant to standard therapy who experienced a decrease in frequency and intensity of tics within days of starting donepezil, and maintained improvement in both tics and attention-related symptoms for 8 months. Because dopamine is known to inhibit acetylcholine interneurons in the striatum, a relationship evident in extrapyramidal syndrome as a result of dopamine blockade by neuroleptics, these reports suggest that acetyl choline may reciprocally decrease dopamine tone and thus improve sometics [41].

\section{Cholinesterase Inhibitors in Other Disorders}

Lanctot et al. [42] reported on a case series of seven patients with dementia with Lewy bodies. These often neuroleptic-resistant patients with significant behavioral disturbances were treated with donepezil in an open label basis. Three patients demonstrated marked improvement as measured by the NPI and two more had improved minimally. Four patients did not complete the intended 8-week treatment period due to side effects or lack of response.

Wengel et al. [43] reported on a case of delirium complicating a preexisting dementia that resolved rapidly following initiation of donepezil. Because cholinergic dysfunction may play a role in the etiology of delirium a trial of donepezil may be appropriate in cases of unknown etiology.

In another anecdotal report, Kishnani et al. [44] described four patients with Down's syndrome treated for an average duration of therapy with donepezil of about 9 months. The foll owing were observed: improvements in communication, expressive language, attention, mood stability, socialization, and adaptive behavior.

Green et al. [45] conducted a 12-week, open-pilot study in 17 patients with multiplesclerosis and cognitive impairment to assess the efficacy and tolerability of donepezil, and reported significant improvement in behavior, attention, memory, and executivefunctioning.

Chol inesterase Inhibitors as Diagnostic Tools Abnormalities of CNS cholinergic tone have been found in a variety of psychiatric disorders [46]. The possibility that such abnormalities may constitute a biological marker and lend themselves to a quick, noni nvasive, and inexpensive test such as the administration of a ChEl, led to a variety of studies in this area.

Major depression is reliably associated with REM sleep abnormalities, suggesting increased cholinergic activation, enhanced dholinergic sensitivity or imbalance in cholinergic and monoaminergic tone. Giles et al. [47•] reported that chol inergic chal lenge using donepezil distinguished between eight depressed patients and eight controls by significantly 
reducing REM sleep latency in depressed patients but not in controls. These results suggest that donepezil can provide a pharmacologic probe of cholinergic tone

Cooney et al. $[48,49]$ examined the specificity of the growth hormone release in response to challenge with the ChEl pyridostigmine in a variety of psychiatric disorders and in heal thy controls. Growth hormone response to the challenge was significantly greater in patients with major depression and panic disorder when compared with heal thy controls. Responses in patients with schizophrenia and al cohol dependence did not differ from the control group. The test demonstrated a sensitivity of $63 \%$ for major depression.

Rubin et al. [50] used physostigmine challenge and measures of subsequent changes in plasma arginine vasopressin (AVP), ACTH, and cortisol to differentiate between groups of patients with major depression. Their results were consistent with a heightened cholinergic sensitivity in premenopausal women, but not in men with major depression.

Steinberg et al. [51] found a significant increase in depressi ve symptoms following physostigmine infusion when compared to placebo in patients with borderline personality disorder (BPD) but not other personality disorders, when compared with normal controls. The study was conducted in 34 patients with personality disorder ( 10 with BPD) and 11 normal controls [51].

\section{Conclusions}

Donepezil as well as other acetylcholinesterase inhibitors appear to have effects on human mood and behavior. Recent years have witnessed a growing number of preliminary observations with thenewly introduced orally administered forms of cholinesterase inhibitors. Applications for cholinesterase inhibitors included both diagnostic and therapeutic interventions. Specifical ly, studies suggest that cholinesterase inhibitors may be useful in the diagnosis of depression and personality disorders, and that they may have mood and behavioral normalizing effects on symptoms such as depression, mania, apathy, delusions, hallucinations, and delirium, in conditions such as bipolar disorder, schizophrenia, Tourette's disorder, and delirium. Donepezil was involved in the largest number of studies and was associated with the largest number of positive results. In view of the established beneficial effect of ChEls on cognition, and considering the close relationship between systems that regulate cognition, mood, and behavior in the CNS, further controlled studies are needed to establish a primary effect of ChEls on mood and behavior and to clarify the diagnostic and therapeutic value of ChEls as pharmacologic modulators of human mood and behavior.
References and Recommended Reading

Papers of particular interest, published recently, have been highlighted as:

- Of importance

- Of major importance

1. Willoughby EF: Pilocarpine in threatening mania. Lancet 1889, i:1030.

2. Bowers MB, Goodman E, Sim VM: Some behavioral changes in man following anticholinesterase administration. J N erv M ent D is 1964, 138:383-389.

3. Grob A, Harvey AM, Langworthy OR, Lilienthal JL: The administration of di-isopropylfluorophosphonate (DFP) to man. Bull Johns H opkins H osp 1947, LXXXI:257-266.

4. Rowntree DW, Nevin S, Wilson A: The effects of diisopropylfluorophosphonate in schizophrenia and manic depressive psychosis. J N eurol N eurosurg Psychiatry 1950, 13:47-59.

5. Janowsky DS, el-Yousef MK, DavisJM, Hubbard B, Sekerke HJ: Cholinergic reversal of manic symptoms. Lancet 1972, 1:1236-1237.

6. Janowsky DS, el-Yousef MK, DavisJM, Sekerke HJ: A cholinergic-adrenergic hypothesis of mania and depression. Lancet 1972, 2:632-635.

7. Safer DJ, Allen RP: The central effects of scopolamine in man. Biol Psychiatry 1971, 3:347-355.

8. Janowsky DS, Risch SC, Kennedy B, et al.: Central muscarinic effects of physostigmine on mood, cardiovascular function, pituitary and adrenal neuroendocrine release. Psychopharmacology (Berl) 1986, 89:150-154.

9. Sitaram N, Nurnberger JI, Jr, Gershon ES, Gillin JC: Cholinergic regulation of mood and REM sleep: potential model and marker of vulnerability to affective disorder. Am J Psychiatry 1982, 139:571-576.

10. Carroll BJ, Frazer A, Schless A, Mendels J: Cholinergic reversal of manic symptoms. Lancet 1973, 1:427-428.

11. Leong SS, Brown WA: Acetylcholine and affective disorder. J N eural Transm 1987, 70:295-312.

12. Davis KL, Berger PA: Pharmacological investigations of the cholinergic imbalance hypotheses of movement disorders and psychosis. Biol Psychiatry 1978, 13:23-49.

13. Geldmacher DS: D onepezil (Aricept) therapy for Alzheimer's disease Compr Ther 1997, 23:492-493.

14. CummingsJL, Mega M, Gray K, et al.: The neuropsychiatric inventory: comprehensive assessment of psychopathology in dementia. N eurology 1994, 44:2308-2314.

15. Weiner MF, Martin-Cook K, Foster BM, et al.: Effects of donepezil on emotional/behavioral symptoms in Alzheimer's disease patients. J Clin Psychiatry 2000, 61:487-492.

16. Cummings JL, Donohue JA, Brooks RL: The relationship between donepezil and behavioral disturbances in patients with Alzheimer's disease. Am J Geriatr Psychiatry 2000, 8:134-140.

17. Frolich L, Berger F, Sramko CA, et al.: Effect of donepezil on behavior and activities of daily living in clinical practice. Alzheimer's Disease and Related Disorders Association World Alzheimer Congress. Washington, DC: July 9-18, 2000.

18. Hecker J, Fon D, Gauthier $\mathrm{S}$, et al.: Benefits of donepezil in the treatment of behavioral problems in moderate to severe Alzheimer's disease. Alzheimer's D isease and Related Disorders Association World Alzheimer Congress. Washington, DC: July 9-18, 2000.

19. Mega MS, Masterman DM, O'Connor SM, et al.: The spectrum of behavioral responses to cholinesterase inhibitor therapy in Alzheimer disease. Arch $N$ eurology 1999, 56:1388-1393.

20. Mega MS, O'Connor SM, Lee L, et al.: O rbital frontal and anterior cingulate pretreatment perfusion defects on $99 \mathrm{mTC}-\mathrm{H}$ M PAO -SPECT are associated with behavioral response to cholinesterase inhibitor therapy in Alzheimer's disease. 50th Annual M eeting of the American Academy of N eurology. Minneapolis, MN: April 25-May 2, 1998 (Neurology 1998, 50(Suppl 4): A250). 
21. - Mega MS, Dinov ID, Lee L, et al.: O rbital and dorsolateral frontal perfusion defect associated with behavioral response to cholinesterase inhibitor therapy in Alzheimer's disease. J N europsych Clinl N eur 2000, 12:209-218.

This study establishes an important association between the administration of $\mathrm{ChEl}$, consequent behavioral changes and corresponding brain imaging findings.

22. - Kaufer D: Beyond the cholinergic hypothesis: the effect of metrifonate and other cholinesterase inhibitors on neuropsychiatric symptoms in Alzheimer's disease. D ement Geriatr Cogn Disord 1998, 9(Suppl 2):8-14.

This study describes the first controlled study of the effect of ChEls on behavior in $A D$ patients.

23. Morris JC, Cyrus PA, Orazem J, et al.: Metrifonate benefits cognitive, behavioral, and global function in patients with Alzheimer's disease. N eurology 1998, 5:1222-1230.

24. CummingsJL, Cyrus PA, Gulanski B, et al.: $M$ etrifonate efficacy in the treatment of psychiatric and behavioral disturbances of Alzheimer's disease patients. 1998 Annual M eeting of the American Geriatrics Society and the American Federation of Aging Research. Seattle, WA: May 6-10, 1998 (J Am Geriatr Soc 1998 46:S65).

25. Raskind MA, Cyrus PA, Ruzicka BB, Gulanski Bl: The effects of metrifonate on the cognitive, behavioral, and functional performance of Alzheimer's disease patients. Metrifonate Study Group. J Clin Psychiatry 1999, 5:318-25.

26. Becker RE, Colliver JA, Markwell SJ, et al.: Effects of metrifonate on cognitive decline in Alzheimer disease: a doubleblind, placebo-controlled, 6-month study. Alzheimer D is Assoc D isord 1998, 1:4-7.

27. Raskind MA, Sadowsky CH, Sigmund WR, et al.: Effect of tacrine on language, praxis, and noncognitive behavioral problems in Alzheimer disease. Arch N eurol 1997, 54:836-40.

28. Rosler M, Anand R, Cicin-Sain A, et al.: Efficacy and safety of rivastigmine in patients with Alzheimer's disease: international randomised controlled trial. Br M ed J 1999, 318:633-638.

29. Tariot PN, Solomon PR, MorrisJC, et al.: A 5-month, randomized, placebo-controlled trial of galantamine in $A D$. The Galantamine USA-10 Study Group. N eurology 2000, 54:2269-2276.

30. Raskind MA, Peskind ER, Wessel T, Yuan W: Galantamine in AD: A 6-month randomized, placebo-controlled trial with a 6-month extension. The Galantamine USA-1 Study Group. N eurology 2000, 54:2261-2268.

31. Thal LJ, Ferguson JM, Mintzer J, Raskin A, Targum SD: A 24-week randomized trial of controlled-release physostigmine in patients with Alzheimer's disease. N eurology 1999, 52:1146- 11152.

32. van Dyck $\mathrm{CH}$, Newhouse P, Falk WE, Mattes JA: Extendedrelease physostigmine in Alzheimer disease: a multicenter, double-blind, 12-week study with dose enrichment. Physostigmine Study Group. Arch Gen Psychiatry 2000, 57:157-164.

33. Burt T, Prudic J, Peyser S, et al.: Learning and memory in bipolar and unipolar major depression: effects of aging. N europsychiatry N europhysiol Behav N eurol 2000, in press.

34. - Burt T, Sachs GS, Demopulos C: Donepezil in treatmentresistant bipolar disorder. Biol Psychiatry 1999, 45:959-964.

This is the first report on the use of the new, orally administered, ChEls in mood disorders. Donepezil hydrochloride was given to 11 treatment-resistant patients with bipolar disorder on an open-label basis. Six patients exhibited a 2 or more improvement on the CGI severity scale over a 6-week period with five patients improving within the first 2 weeks.
35. Goodnick PJ, Fieve RR, Peselow ED, et al.: Double-blind treatment of major depression with fluoxetine: use of pattern analysis and relation of HAM-D score to CGI change. Psychopharmacol Bull 1987, 23:162-163.

36. Risch SC, Nahas Z, Horner MD, et al.: Donepezil augmentation of antipsychotics in schizophrenia: cognitive and fMRI effects. Biological Psychiatry 2000, 47:S17-S18.

37. Bergman J, Brettholz I: Beneficial effects of donepezil (M emorit) on psychotic exacerbation in a schizophrenic patient with severe cognitive decline. 22nd Collegian International N europsychopharmacology Congress. Brussels, Belgium: July 9-13, 2000 (Int J Neuropsychopharmacol 2000, 3(Suppl 1): S167).

38. Schwarzkopf SB, Lamberti JS, Pierce D, et al.: Treatment of refractory cognitive and negative symptoms of schizophrenia with donepezil: a case series. 7th International Congress on Schizophrenia Research. Santa Fe, NM: April 17-21, 1999 (Schizophren Res 1999, 36(1-3):297).

39. Burke WJ, Roccaforte WH, Wengel SP: Treating visual hallucinations with donepezil. Am J Psychiatry 1999, 156:1117-1118.

40. Juncos JL, Roberts VJ et al.: C holinergic strategies in Tourette syndrome: an open-label trial of tacrine hydrochloride. American Academy of N eurology 49th Annual M eeting Program. Boston, MA: April 12-19, 1997 (Neurology 1997, 48(3 Suppl 2):A397).

41. Hoopes SP: Donepezil for Tourette's disorder and AD HD. J Clin Psychopharmacol 1999, 4:381-382.

42. Lanctot $K L$, Herrmann N: Donepezil for behavioural disorders associated with Lewy bodies: a case series. Int J Geriatr Psychiatry 2000, 15:338-345.

43. Wengel SP, Roccaforte WH, Burke WJ: Donepezil improves symptoms of delirium in dementia: implications for future research. J Geriatr Psychiatry N eurol 1998, 11:159-161.

44. Kishnani PS, Sullivan JA, Walter BK, et al.: Cholinergic therapy for D own's syndrome Lancet 1999, 353:1064-1065.

45. Greene YM, Tariot PN, Wishart H, et al.: A 12-week, open trial of donepezil hydrochloride in patients with multiple sclerosis and associated cognitive impairments. J Clin Psychopharmacol 2000, 20:350-356.

46. Janowsky DS, Overstreet DH, Nurnberger JI, Jr: Is cholinergic sensitivity a genetic marker for the affective disorders? Am J M ed Genet 1994, 54:335-344.

47. - Giles DE, Perlis ML, Orff HJ, et al.: New cholinergic test of sleep/mood dysregulation in familial depression. Biol Psychiatry 2000, 47:S85.

This study suggests that donepezil may be used as a marker of cholinergic tone in the central nervous system.

48. Cooney JM, Lucey JV, O'Keane V, Dinan TG: Specificity of the pyridostigmine/growth hormone challenge in the diagnosis of depression. Biol Psychiatry 1997, 42:827-833.

49. Cooney JM, Lucey JV, Dinan TG: Enhanced growth hormone responses to pyridostigmine challenge in patients with panic disorder. Br J Psychiatry 1997, 170:159-161.

50. Rubin RT, O'Toole SM, Rhodes ME, et al.: H ypothalamo-pituitary-adrenal cortical responses to low-dose physostigmine and arginine vasopressin administration: sex differences between major depressives and matched control subjects. Psychiatry Res 1999, 89:1-20.

51. Steinberg BJ, Trestman R, Mitropoulou V, et al.: Depressive response to physostigmine challenge in borderline personality disorder patients. N europsychopharmacology 1997, 17:264-273. 